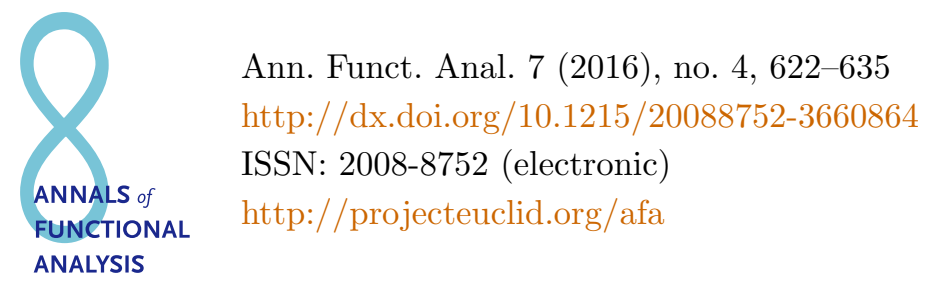

\title{
ON THE ARAKI-LIEB-THIRRING INEQUALITY IN THE SEMIFINITE VON NEUMANN ALGEBRA
}

\author{
YAZHOU HAN
}

Communicated by J.-C. Bourin

\begin{abstract}
This paper extends a recent matrix trace inequality of BourinLee to semifinite von Neumann algebras. This provides a generalization of the Lieb-Thirring-type inequality in von Neumann algebras due to Kosaki. Some new inequalities, even in the matrix case, are also given for the Heinz means.
\end{abstract}

\section{INTRODUCTION}

Let $\mathbb{M}_{n}$ be the space of $n \times n$ complex matrices. The Lieb-Thirring inequality [17] states that, for $0 \leq A, B \in \mathbb{M}_{n}$ and $p \geq 1$,

$$
\operatorname{Tr}\left(\left(B^{\frac{1}{2}} A B^{\frac{1}{2}}\right)^{p}\right) \leq \operatorname{Tr}\left(A^{p} B^{p}\right) .
$$

Let $A$ and $B$ be positive self-adjoint operators on a Hilbert space, and let $f$ be any increasing continuous function on $[0, \infty)$ such that $f(0)=0$ and $t \rightarrow f\left(e^{t}\right)$ is a convex function. Araki [2] shows a refinement of the Lieb-Thirring inequality as follows:

$$
\operatorname{Tr} f\left(\left(B^{\frac{1}{2}} A B^{\frac{1}{2}}\right)^{q}\right) \leq \operatorname{Tr} f\left(B^{\frac{q}{2}} A^{q} B^{\frac{q}{2}}\right) \quad \text { for all } q \geq 1 .
$$

Here the condition $f(0)=0$ ensures that the trace is well defined; that is, $\infty-\infty$ does not occur. Recently, Bourin and Lee [6] proved that if $f$ is increasing and $t \rightarrow f\left(e^{t}\right)$ is convex, then the inequality

$$
\operatorname{Tr} f\left(\left(B Z^{*} A Z B\right)^{q}\right) \leq \operatorname{Tr} f\left(B^{q} Z^{*} A^{q} Z B^{q}\right) \quad \text { for all } q \geq 1
$$

Copyright 2016 by the Tusi Mathematical Research Group.

Received Dec. 15, 2015; Accepted May 5, 2016.

2010 Mathematics Subject Classification. Primary 47A63; Secondary 46L52.

Keywords. Araki-Lieb-Thirring inequality, von Neumann algebra, $\tau$-measurable operator. 


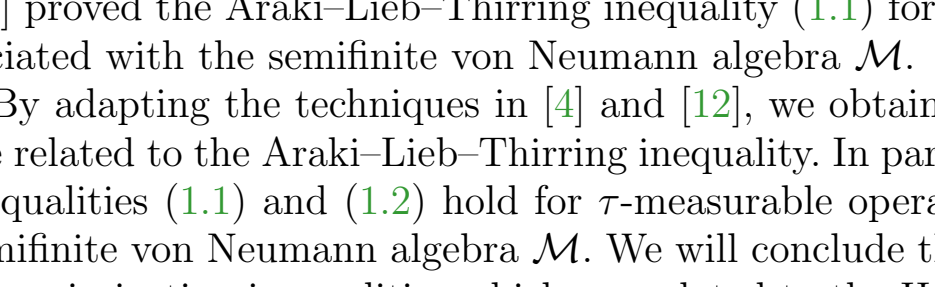

\title{
ON THE ARAKI-LIEB-THIRRING INEQUALITY IN THE SEMIFINITE VON NEUMANN ALGEBRA
}

\author{
YAZHOU HAN
}

Communicated by J.-C. Bourin

\begin{abstract}
This paper extends a recent matrix trace inequality of BourinLee to semifinite von Neumann algebras. This provides a generalization of the Lieb-Thirring-type inequality in von Neumann algebras due to Kosaki. Some new inequalities, even in the matrix case, are also given for the Heinz means.
\end{abstract}

\section{INTRODUCTION}

Let $\mathbb{M}_{n}$ be the space of $n \times n$ complex matrices. The Lieb-Thirring inequality [17] states that, for $0 \leq A, B \in \mathbb{M}_{n}$ and $p \geq 1$,

$$
\operatorname{Tr}\left(\left(B^{\frac{1}{2}} A B^{\frac{1}{2}}\right)^{p}\right) \leq \operatorname{Tr}\left(A^{p} B^{p}\right) .
$$

Let $A$ and $B$ be positive self-adjoint operators on a Hilbert space, and let $f$ be any increasing continuous function on $[0, \infty)$ such that $f(0)=0$ and $t \rightarrow f\left(e^{t}\right)$ is a convex function. Araki [2] shows a refinement of the Lieb-Thirring inequality as follows:

$$
\operatorname{Tr} f\left(\left(B^{\frac{1}{2}} A B^{\frac{1}{2}}\right)^{q}\right) \leq \operatorname{Tr} f\left(B^{\frac{q}{2}} A^{q} B^{\frac{q}{2}}\right) \quad \text { for all } q \geq 1 .
$$

Here the condition $f(0)=0$ ensures that the trace is well defined; that is, $\infty-\infty$ does not occur. Recently, Bourin and Lee [6] proved that if $f$ is increasing and $t \rightarrow f\left(e^{t}\right)$ is convex, then the inequality

$$
\operatorname{Tr} f\left(\left(B Z^{*} A Z B\right)^{q}\right) \leq \operatorname{Tr} f\left(B^{q} Z^{*} A^{q} Z B^{q}\right) \quad \text { for all } q \geq 1
$$

Copyright 2016 by the Tusi Mathematical Research Group.

Received Dec. 15, 2015; Accepted May 5, 2016.

2010 Mathematics Subject Classification. Primary 47A63; Secondary 46L52.

Keywords. Araki-Lieb-Thirring inequality, von Neumann algebra, $\tau$-measurable operator. 


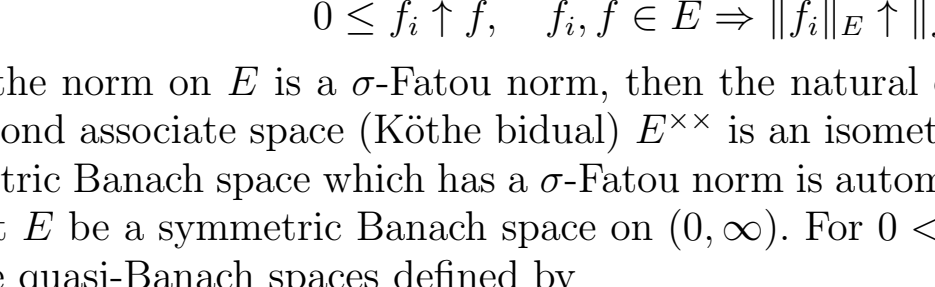

\title{
ON THE ARAKI-LIEB-THIRRING INEQUALITY IN THE SEMIFINITE VON NEUMANN ALGEBRA
}

\author{
YAZHOU HAN
}

Communicated by J.-C. Bourin

\begin{abstract}
This paper extends a recent matrix trace inequality of BourinLee to semifinite von Neumann algebras. This provides a generalization of the Lieb-Thirring-type inequality in von Neumann algebras due to Kosaki. Some new inequalities, even in the matrix case, are also given for the Heinz means.
\end{abstract}

\section{INTRODUCTION}

Let $\mathbb{M}_{n}$ be the space of $n \times n$ complex matrices. The Lieb-Thirring inequality [17] states that, for $0 \leq A, B \in \mathbb{M}_{n}$ and $p \geq 1$,

$$
\operatorname{Tr}\left(\left(B^{\frac{1}{2}} A B^{\frac{1}{2}}\right)^{p}\right) \leq \operatorname{Tr}\left(A^{p} B^{p}\right) .
$$

Let $A$ and $B$ be positive self-adjoint operators on a Hilbert space, and let $f$ be any increasing continuous function on $[0, \infty)$ such that $f(0)=0$ and $t \rightarrow f\left(e^{t}\right)$ is a convex function. Araki [2] shows a refinement of the Lieb-Thirring inequality as follows:

$$
\operatorname{Tr} f\left(\left(B^{\frac{1}{2}} A B^{\frac{1}{2}}\right)^{q}\right) \leq \operatorname{Tr} f\left(B^{\frac{q}{2}} A^{q} B^{\frac{q}{2}}\right) \quad \text { for all } q \geq 1 .
$$

Here the condition $f(0)=0$ ensures that the trace is well defined; that is, $\infty-\infty$ does not occur. Recently, Bourin and Lee [6] proved that if $f$ is increasing and $t \rightarrow f\left(e^{t}\right)$ is convex, then the inequality

$$
\operatorname{Tr} f\left(\left(B Z^{*} A Z B\right)^{q}\right) \leq \operatorname{Tr} f\left(B^{q} Z^{*} A^{q} Z B^{q}\right) \quad \text { for all } q \geq 1
$$

Copyright 2016 by the Tusi Mathematical Research Group.

Received Dec. 15, 2015; Accepted May 5, 2016.

2010 Mathematics Subject Classification. Primary 47A63; Secondary 46L52.

Keywords. Araki-Lieb-Thirring inequality, von Neumann algebra, $\tau$-measurable operator. 


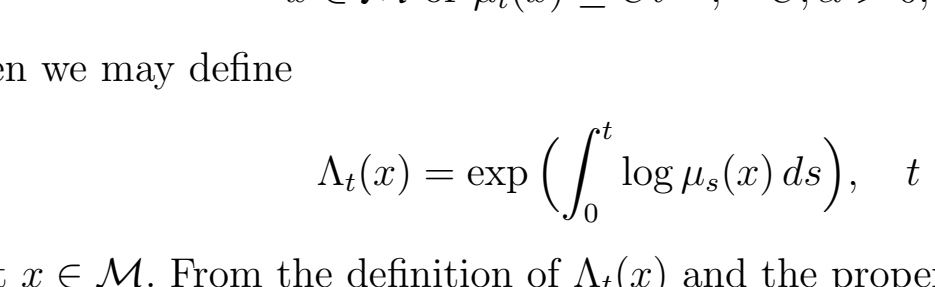

\title{
ON THE ARAKI-LIEB-THIRRING INEQUALITY IN THE SEMIFINITE VON NEUMANN ALGEBRA
}

\author{
YAZHOU HAN
}

Communicated by J.-C. Bourin

\begin{abstract}
This paper extends a recent matrix trace inequality of BourinLee to semifinite von Neumann algebras. This provides a generalization of the Lieb-Thirring-type inequality in von Neumann algebras due to Kosaki. Some new inequalities, even in the matrix case, are also given for the Heinz means.
\end{abstract}

\section{INTRODUCTION}

Let $\mathbb{M}_{n}$ be the space of $n \times n$ complex matrices. The Lieb-Thirring inequality [17] states that, for $0 \leq A, B \in \mathbb{M}_{n}$ and $p \geq 1$,

$$
\operatorname{Tr}\left(\left(B^{\frac{1}{2}} A B^{\frac{1}{2}}\right)^{p}\right) \leq \operatorname{Tr}\left(A^{p} B^{p}\right) .
$$

Let $A$ and $B$ be positive self-adjoint operators on a Hilbert space, and let $f$ be any increasing continuous function on $[0, \infty)$ such that $f(0)=0$ and $t \rightarrow f\left(e^{t}\right)$ is a convex function. Araki [2] shows a refinement of the Lieb-Thirring inequality as follows:

$$
\operatorname{Tr} f\left(\left(B^{\frac{1}{2}} A B^{\frac{1}{2}}\right)^{q}\right) \leq \operatorname{Tr} f\left(B^{\frac{q}{2}} A^{q} B^{\frac{q}{2}}\right) \quad \text { for all } q \geq 1 .
$$

Here the condition $f(0)=0$ ensures that the trace is well defined; that is, $\infty-\infty$ does not occur. Recently, Bourin and Lee [6] proved that if $f$ is increasing and $t \rightarrow f\left(e^{t}\right)$ is convex, then the inequality

$$
\operatorname{Tr} f\left(\left(B Z^{*} A Z B\right)^{q}\right) \leq \operatorname{Tr} f\left(B^{q} Z^{*} A^{q} Z B^{q}\right) \quad \text { for all } q \geq 1
$$

Copyright 2016 by the Tusi Mathematical Research Group.

Received Dec. 15, 2015; Accepted May 5, 2016.

2010 Mathematics Subject Classification. Primary 47A63; Secondary 46L52.

Keywords. Araki-Lieb-Thirring inequality, von Neumann algebra, $\tau$-measurable operator. 


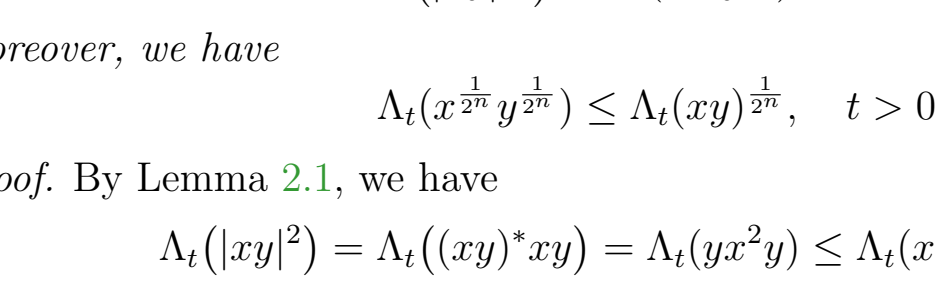

\title{
ON THE ARAKI-LIEB-THIRRING INEQUALITY IN THE SEMIFINITE VON NEUMANN ALGEBRA
}

\author{
YAZHOU HAN
}

Communicated by J.-C. Bourin

\begin{abstract}
This paper extends a recent matrix trace inequality of BourinLee to semifinite von Neumann algebras. This provides a generalization of the Lieb-Thirring-type inequality in von Neumann algebras due to Kosaki. Some new inequalities, even in the matrix case, are also given for the Heinz means.
\end{abstract}

\section{INTRODUCTION}

Let $\mathbb{M}_{n}$ be the space of $n \times n$ complex matrices. The Lieb-Thirring inequality [17] states that, for $0 \leq A, B \in \mathbb{M}_{n}$ and $p \geq 1$,

$$
\operatorname{Tr}\left(\left(B^{\frac{1}{2}} A B^{\frac{1}{2}}\right)^{p}\right) \leq \operatorname{Tr}\left(A^{p} B^{p}\right) .
$$

Let $A$ and $B$ be positive self-adjoint operators on a Hilbert space, and let $f$ be any increasing continuous function on $[0, \infty)$ such that $f(0)=0$ and $t \rightarrow f\left(e^{t}\right)$ is a convex function. Araki [2] shows a refinement of the Lieb-Thirring inequality as follows:

$$
\operatorname{Tr} f\left(\left(B^{\frac{1}{2}} A B^{\frac{1}{2}}\right)^{q}\right) \leq \operatorname{Tr} f\left(B^{\frac{q}{2}} A^{q} B^{\frac{q}{2}}\right) \quad \text { for all } q \geq 1 .
$$

Here the condition $f(0)=0$ ensures that the trace is well defined; that is, $\infty-\infty$ does not occur. Recently, Bourin and Lee [6] proved that if $f$ is increasing and $t \rightarrow f\left(e^{t}\right)$ is convex, then the inequality

$$
\operatorname{Tr} f\left(\left(B Z^{*} A Z B\right)^{q}\right) \leq \operatorname{Tr} f\left(B^{q} Z^{*} A^{q} Z B^{q}\right) \quad \text { for all } q \geq 1
$$

Copyright 2016 by the Tusi Mathematical Research Group.

Received Dec. 15, 2015; Accepted May 5, 2016.

2010 Mathematics Subject Classification. Primary 47A63; Secondary 46L52.

Keywords. Araki-Lieb-Thirring inequality, von Neumann algebra, $\tau$-measurable operator. 


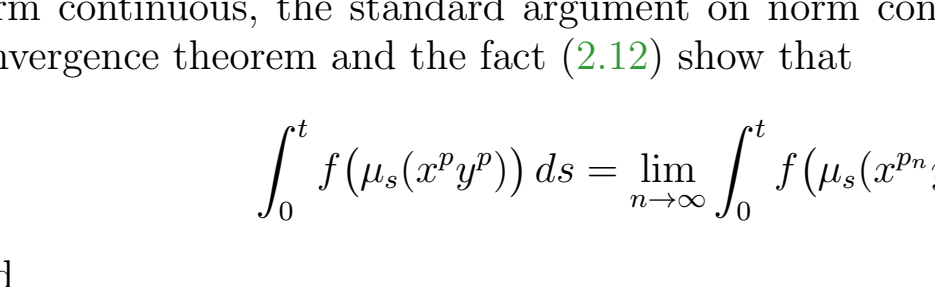

\title{
ON THE ARAKI-LIEB-THIRRING INEQUALITY IN THE SEMIFINITE VON NEUMANN ALGEBRA
}

\author{
YAZHOU HAN
}

Communicated by J.-C. Bourin

\begin{abstract}
This paper extends a recent matrix trace inequality of BourinLee to semifinite von Neumann algebras. This provides a generalization of the Lieb-Thirring-type inequality in von Neumann algebras due to Kosaki. Some new inequalities, even in the matrix case, are also given for the Heinz means.
\end{abstract}

\section{INTRODUCTION}

Let $\mathbb{M}_{n}$ be the space of $n \times n$ complex matrices. The Lieb-Thirring inequality [17] states that, for $0 \leq A, B \in \mathbb{M}_{n}$ and $p \geq 1$,

$$
\operatorname{Tr}\left(\left(B^{\frac{1}{2}} A B^{\frac{1}{2}}\right)^{p}\right) \leq \operatorname{Tr}\left(A^{p} B^{p}\right) .
$$

Let $A$ and $B$ be positive self-adjoint operators on a Hilbert space, and let $f$ be any increasing continuous function on $[0, \infty)$ such that $f(0)=0$ and $t \rightarrow f\left(e^{t}\right)$ is a convex function. Araki [2] shows a refinement of the Lieb-Thirring inequality as follows:

$$
\operatorname{Tr} f\left(\left(B^{\frac{1}{2}} A B^{\frac{1}{2}}\right)^{q}\right) \leq \operatorname{Tr} f\left(B^{\frac{q}{2}} A^{q} B^{\frac{q}{2}}\right) \quad \text { for all } q \geq 1 .
$$

Here the condition $f(0)=0$ ensures that the trace is well defined; that is, $\infty-\infty$ does not occur. Recently, Bourin and Lee [6] proved that if $f$ is increasing and $t \rightarrow f\left(e^{t}\right)$ is convex, then the inequality

$$
\operatorname{Tr} f\left(\left(B Z^{*} A Z B\right)^{q}\right) \leq \operatorname{Tr} f\left(B^{q} Z^{*} A^{q} Z B^{q}\right) \quad \text { for all } q \geq 1
$$

Copyright 2016 by the Tusi Mathematical Research Group.

Received Dec. 15, 2015; Accepted May 5, 2016.

2010 Mathematics Subject Classification. Primary 47A63; Secondary 46L52.

Keywords. Araki-Lieb-Thirring inequality, von Neumann algebra, $\tau$-measurable operator. 


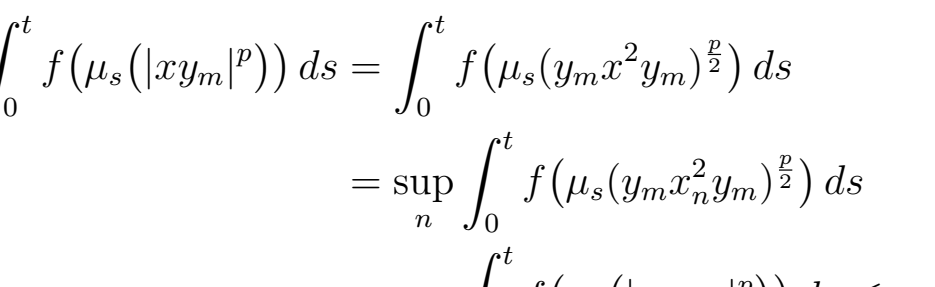

\title{
ON THE ARAKI-LIEB-THIRRING INEQUALITY IN THE SEMIFINITE VON NEUMANN ALGEBRA
}

\author{
YAZHOU HAN
}

Communicated by J.-C. Bourin

\begin{abstract}
This paper extends a recent matrix trace inequality of BourinLee to semifinite von Neumann algebras. This provides a generalization of the Lieb-Thirring-type inequality in von Neumann algebras due to Kosaki. Some new inequalities, even in the matrix case, are also given for the Heinz means.
\end{abstract}

\section{INTRODUCTION}

Let $\mathbb{M}_{n}$ be the space of $n \times n$ complex matrices. The Lieb-Thirring inequality [17] states that, for $0 \leq A, B \in \mathbb{M}_{n}$ and $p \geq 1$,

$$
\operatorname{Tr}\left(\left(B^{\frac{1}{2}} A B^{\frac{1}{2}}\right)^{p}\right) \leq \operatorname{Tr}\left(A^{p} B^{p}\right) .
$$

Let $A$ and $B$ be positive self-adjoint operators on a Hilbert space, and let $f$ be any increasing continuous function on $[0, \infty)$ such that $f(0)=0$ and $t \rightarrow f\left(e^{t}\right)$ is a convex function. Araki [2] shows a refinement of the Lieb-Thirring inequality as follows:

$$
\operatorname{Tr} f\left(\left(B^{\frac{1}{2}} A B^{\frac{1}{2}}\right)^{q}\right) \leq \operatorname{Tr} f\left(B^{\frac{q}{2}} A^{q} B^{\frac{q}{2}}\right) \quad \text { for all } q \geq 1 .
$$

Here the condition $f(0)=0$ ensures that the trace is well defined; that is, $\infty-\infty$ does not occur. Recently, Bourin and Lee [6] proved that if $f$ is increasing and $t \rightarrow f\left(e^{t}\right)$ is convex, then the inequality

$$
\operatorname{Tr} f\left(\left(B Z^{*} A Z B\right)^{q}\right) \leq \operatorname{Tr} f\left(B^{q} Z^{*} A^{q} Z B^{q}\right) \quad \text { for all } q \geq 1
$$

Copyright 2016 by the Tusi Mathematical Research Group.

Received Dec. 15, 2015; Accepted May 5, 2016.

2010 Mathematics Subject Classification. Primary 47A63; Secondary 46L52.

Keywords. Araki-Lieb-Thirring inequality, von Neumann algebra, $\tau$-measurable operator. 


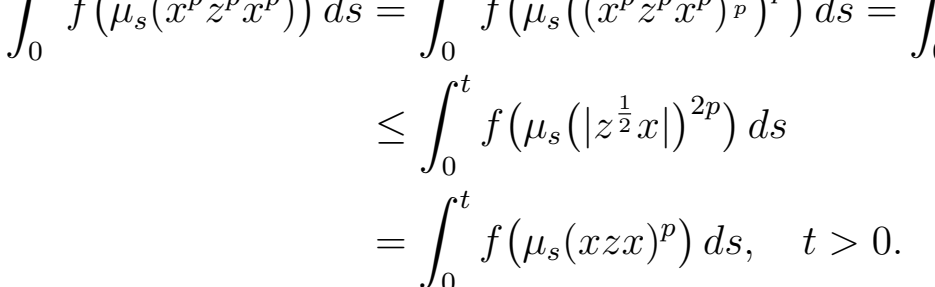

\title{
ON THE ARAKI-LIEB-THIRRING INEQUALITY IN THE SEMIFINITE VON NEUMANN ALGEBRA
}

\author{
YAZHOU HAN
}

Communicated by J.-C. Bourin

\begin{abstract}
This paper extends a recent matrix trace inequality of BourinLee to semifinite von Neumann algebras. This provides a generalization of the Lieb-Thirring-type inequality in von Neumann algebras due to Kosaki. Some new inequalities, even in the matrix case, are also given for the Heinz means.
\end{abstract}

\section{INTRODUCTION}

Let $\mathbb{M}_{n}$ be the space of $n \times n$ complex matrices. The Lieb-Thirring inequality [17] states that, for $0 \leq A, B \in \mathbb{M}_{n}$ and $p \geq 1$,

$$
\operatorname{Tr}\left(\left(B^{\frac{1}{2}} A B^{\frac{1}{2}}\right)^{p}\right) \leq \operatorname{Tr}\left(A^{p} B^{p}\right) .
$$

Let $A$ and $B$ be positive self-adjoint operators on a Hilbert space, and let $f$ be any increasing continuous function on $[0, \infty)$ such that $f(0)=0$ and $t \rightarrow f\left(e^{t}\right)$ is a convex function. Araki [2] shows a refinement of the Lieb-Thirring inequality as follows:

$$
\operatorname{Tr} f\left(\left(B^{\frac{1}{2}} A B^{\frac{1}{2}}\right)^{q}\right) \leq \operatorname{Tr} f\left(B^{\frac{q}{2}} A^{q} B^{\frac{q}{2}}\right) \quad \text { for all } q \geq 1 .
$$

Here the condition $f(0)=0$ ensures that the trace is well defined; that is, $\infty-\infty$ does not occur. Recently, Bourin and Lee [6] proved that if $f$ is increasing and $t \rightarrow f\left(e^{t}\right)$ is convex, then the inequality

$$
\operatorname{Tr} f\left(\left(B Z^{*} A Z B\right)^{q}\right) \leq \operatorname{Tr} f\left(B^{q} Z^{*} A^{q} Z B^{q}\right) \quad \text { for all } q \geq 1
$$

Copyright 2016 by the Tusi Mathematical Research Group.

Received Dec. 15, 2015; Accepted May 5, 2016.

2010 Mathematics Subject Classification. Primary 47A63; Secondary 46L52.

Keywords. Araki-Lieb-Thirring inequality, von Neumann algebra, $\tau$-measurable operator. 


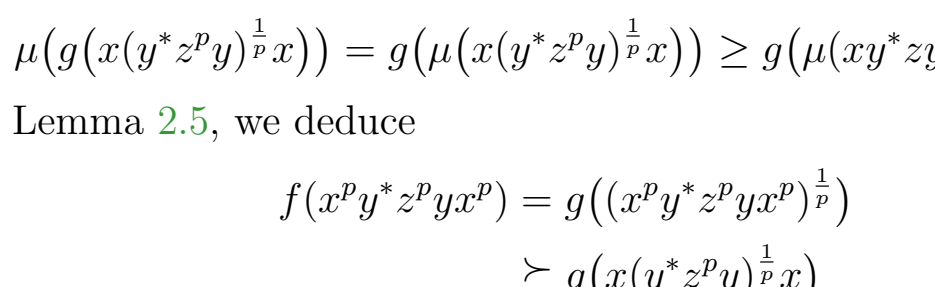

\title{
ON THE ARAKI-LIEB-THIRRING INEQUALITY IN THE SEMIFINITE VON NEUMANN ALGEBRA
}

\author{
YAZHOU HAN
}

Communicated by J.-C. Bourin

\begin{abstract}
This paper extends a recent matrix trace inequality of BourinLee to semifinite von Neumann algebras. This provides a generalization of the Lieb-Thirring-type inequality in von Neumann algebras due to Kosaki. Some new inequalities, even in the matrix case, are also given for the Heinz means.
\end{abstract}

\section{INTRODUCTION}

Let $\mathbb{M}_{n}$ be the space of $n \times n$ complex matrices. The Lieb-Thirring inequality [17] states that, for $0 \leq A, B \in \mathbb{M}_{n}$ and $p \geq 1$,

$$
\operatorname{Tr}\left(\left(B^{\frac{1}{2}} A B^{\frac{1}{2}}\right)^{p}\right) \leq \operatorname{Tr}\left(A^{p} B^{p}\right) .
$$

Let $A$ and $B$ be positive self-adjoint operators on a Hilbert space, and let $f$ be any increasing continuous function on $[0, \infty)$ such that $f(0)=0$ and $t \rightarrow f\left(e^{t}\right)$ is a convex function. Araki [2] shows a refinement of the Lieb-Thirring inequality as follows:

$$
\operatorname{Tr} f\left(\left(B^{\frac{1}{2}} A B^{\frac{1}{2}}\right)^{q}\right) \leq \operatorname{Tr} f\left(B^{\frac{q}{2}} A^{q} B^{\frac{q}{2}}\right) \quad \text { for all } q \geq 1 .
$$

Here the condition $f(0)=0$ ensures that the trace is well defined; that is, $\infty-\infty$ does not occur. Recently, Bourin and Lee [6] proved that if $f$ is increasing and $t \rightarrow f\left(e^{t}\right)$ is convex, then the inequality

$$
\operatorname{Tr} f\left(\left(B Z^{*} A Z B\right)^{q}\right) \leq \operatorname{Tr} f\left(B^{q} Z^{*} A^{q} Z B^{q}\right) \quad \text { for all } q \geq 1
$$

Copyright 2016 by the Tusi Mathematical Research Group.

Received Dec. 15, 2015; Accepted May 5, 2016.

2010 Mathematics Subject Classification. Primary 47A63; Secondary 46L52.

Keywords. Araki-Lieb-Thirring inequality, von Neumann algebra, $\tau$-measurable operator. 


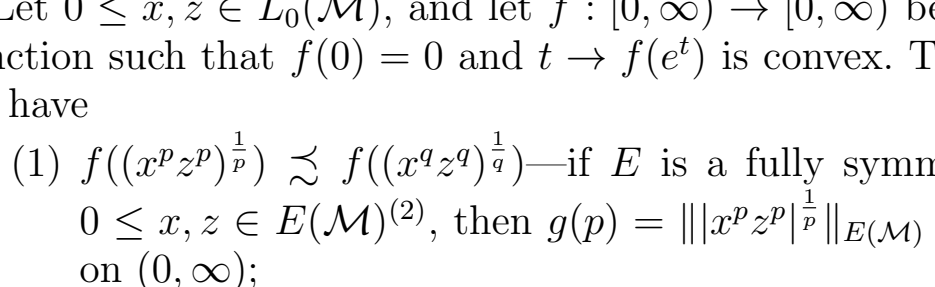

\title{
ON THE ARAKI-LIEB-THIRRING INEQUALITY IN THE SEMIFINITE VON NEUMANN ALGEBRA
}

\author{
YAZHOU HAN
}

Communicated by J.-C. Bourin

\begin{abstract}
This paper extends a recent matrix trace inequality of BourinLee to semifinite von Neumann algebras. This provides a generalization of the Lieb-Thirring-type inequality in von Neumann algebras due to Kosaki. Some new inequalities, even in the matrix case, are also given for the Heinz means.
\end{abstract}

\section{INTRODUCTION}

Let $\mathbb{M}_{n}$ be the space of $n \times n$ complex matrices. The Lieb-Thirring inequality [17] states that, for $0 \leq A, B \in \mathbb{M}_{n}$ and $p \geq 1$,

$$
\operatorname{Tr}\left(\left(B^{\frac{1}{2}} A B^{\frac{1}{2}}\right)^{p}\right) \leq \operatorname{Tr}\left(A^{p} B^{p}\right) .
$$

Let $A$ and $B$ be positive self-adjoint operators on a Hilbert space, and let $f$ be any increasing continuous function on $[0, \infty)$ such that $f(0)=0$ and $t \rightarrow f\left(e^{t}\right)$ is a convex function. Araki [2] shows a refinement of the Lieb-Thirring inequality as follows:

$$
\operatorname{Tr} f\left(\left(B^{\frac{1}{2}} A B^{\frac{1}{2}}\right)^{q}\right) \leq \operatorname{Tr} f\left(B^{\frac{q}{2}} A^{q} B^{\frac{q}{2}}\right) \quad \text { for all } q \geq 1 .
$$

Here the condition $f(0)=0$ ensures that the trace is well defined; that is, $\infty-\infty$ does not occur. Recently, Bourin and Lee [6] proved that if $f$ is increasing and $t \rightarrow f\left(e^{t}\right)$ is convex, then the inequality

$$
\operatorname{Tr} f\left(\left(B Z^{*} A Z B\right)^{q}\right) \leq \operatorname{Tr} f\left(B^{q} Z^{*} A^{q} Z B^{q}\right) \quad \text { for all } q \geq 1
$$

Copyright 2016 by the Tusi Mathematical Research Group.

Received Dec. 15, 2015; Accepted May 5, 2016.

2010 Mathematics Subject Classification. Primary 47A63; Secondary 46L52.

Keywords. Araki-Lieb-Thirring inequality, von Neumann algebra, $\tau$-measurable operator. 


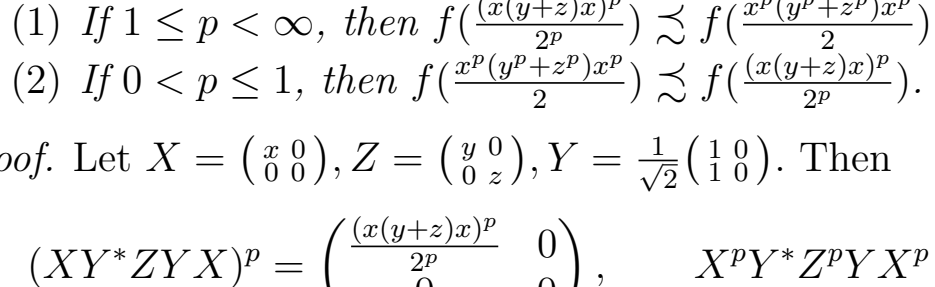

\title{
ON THE ARAKI-LIEB-THIRRING INEQUALITY IN THE SEMIFINITE VON NEUMANN ALGEBRA
}

\author{
YAZHOU HAN
}

Communicated by J.-C. Bourin

\begin{abstract}
This paper extends a recent matrix trace inequality of BourinLee to semifinite von Neumann algebras. This provides a generalization of the Lieb-Thirring-type inequality in von Neumann algebras due to Kosaki. Some new inequalities, even in the matrix case, are also given for the Heinz means.
\end{abstract}

\section{INTRODUCTION}

Let $\mathbb{M}_{n}$ be the space of $n \times n$ complex matrices. The Lieb-Thirring inequality [17] states that, for $0 \leq A, B \in \mathbb{M}_{n}$ and $p \geq 1$,

$$
\operatorname{Tr}\left(\left(B^{\frac{1}{2}} A B^{\frac{1}{2}}\right)^{p}\right) \leq \operatorname{Tr}\left(A^{p} B^{p}\right) .
$$

Let $A$ and $B$ be positive self-adjoint operators on a Hilbert space, and let $f$ be any increasing continuous function on $[0, \infty)$ such that $f(0)=0$ and $t \rightarrow f\left(e^{t}\right)$ is a convex function. Araki [2] shows a refinement of the Lieb-Thirring inequality as follows:

$$
\operatorname{Tr} f\left(\left(B^{\frac{1}{2}} A B^{\frac{1}{2}}\right)^{q}\right) \leq \operatorname{Tr} f\left(B^{\frac{q}{2}} A^{q} B^{\frac{q}{2}}\right) \quad \text { for all } q \geq 1 .
$$

Here the condition $f(0)=0$ ensures that the trace is well defined; that is, $\infty-\infty$ does not occur. Recently, Bourin and Lee [6] proved that if $f$ is increasing and $t \rightarrow f\left(e^{t}\right)$ is convex, then the inequality

$$
\operatorname{Tr} f\left(\left(B Z^{*} A Z B\right)^{q}\right) \leq \operatorname{Tr} f\left(B^{q} Z^{*} A^{q} Z B^{q}\right) \quad \text { for all } q \geq 1
$$

Copyright 2016 by the Tusi Mathematical Research Group.

Received Dec. 15, 2015; Accepted May 5, 2016.

2010 Mathematics Subject Classification. Primary 47A63; Secondary 46L52.

Keywords. Araki-Lieb-Thirring inequality, von Neumann algebra, $\tau$-measurable operator. 


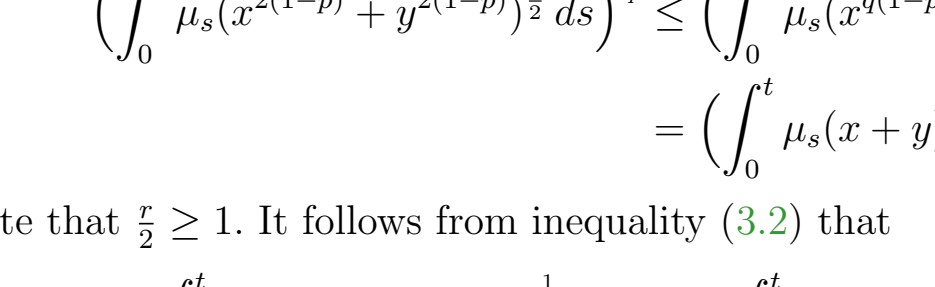

\title{
ON THE ARAKI-LIEB-THIRRING INEQUALITY IN THE SEMIFINITE VON NEUMANN ALGEBRA
}

\author{
YAZHOU HAN
}

Communicated by J.-C. Bourin

\begin{abstract}
This paper extends a recent matrix trace inequality of BourinLee to semifinite von Neumann algebras. This provides a generalization of the Lieb-Thirring-type inequality in von Neumann algebras due to Kosaki. Some new inequalities, even in the matrix case, are also given for the Heinz means.
\end{abstract}

\section{INTRODUCTION}

Let $\mathbb{M}_{n}$ be the space of $n \times n$ complex matrices. The Lieb-Thirring inequality [17] states that, for $0 \leq A, B \in \mathbb{M}_{n}$ and $p \geq 1$,

$$
\operatorname{Tr}\left(\left(B^{\frac{1}{2}} A B^{\frac{1}{2}}\right)^{p}\right) \leq \operatorname{Tr}\left(A^{p} B^{p}\right) .
$$

Let $A$ and $B$ be positive self-adjoint operators on a Hilbert space, and let $f$ be any increasing continuous function on $[0, \infty)$ such that $f(0)=0$ and $t \rightarrow f\left(e^{t}\right)$ is a convex function. Araki [2] shows a refinement of the Lieb-Thirring inequality as follows:

$$
\operatorname{Tr} f\left(\left(B^{\frac{1}{2}} A B^{\frac{1}{2}}\right)^{q}\right) \leq \operatorname{Tr} f\left(B^{\frac{q}{2}} A^{q} B^{\frac{q}{2}}\right) \quad \text { for all } q \geq 1 .
$$

Here the condition $f(0)=0$ ensures that the trace is well defined; that is, $\infty-\infty$ does not occur. Recently, Bourin and Lee [6] proved that if $f$ is increasing and $t \rightarrow f\left(e^{t}\right)$ is convex, then the inequality

$$
\operatorname{Tr} f\left(\left(B Z^{*} A Z B\right)^{q}\right) \leq \operatorname{Tr} f\left(B^{q} Z^{*} A^{q} Z B^{q}\right) \quad \text { for all } q \geq 1
$$

Copyright 2016 by the Tusi Mathematical Research Group.

Received Dec. 15, 2015; Accepted May 5, 2016.

2010 Mathematics Subject Classification. Primary 47A63; Secondary 46L52.

Keywords. Araki-Lieb-Thirring inequality, von Neumann algebra, $\tau$-measurable operator. 


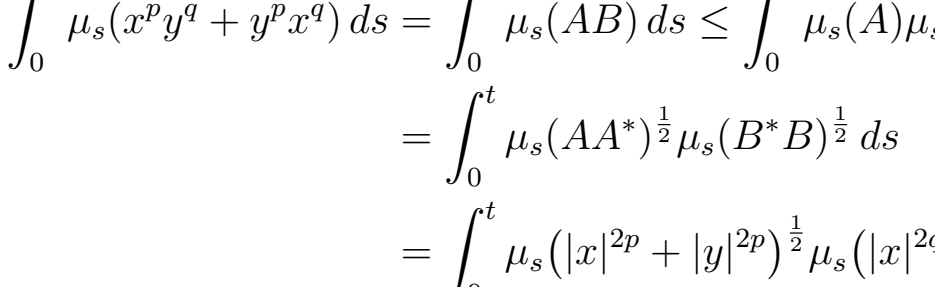

\title{
ON THE ARAKI-LIEB-THIRRING INEQUALITY IN THE SEMIFINITE VON NEUMANN ALGEBRA
}

\author{
YAZHOU HAN
}

Communicated by J.-C. Bourin

\begin{abstract}
This paper extends a recent matrix trace inequality of BourinLee to semifinite von Neumann algebras. This provides a generalization of the Lieb-Thirring-type inequality in von Neumann algebras due to Kosaki. Some new inequalities, even in the matrix case, are also given for the Heinz means.
\end{abstract}

\section{INTRODUCTION}

Let $\mathbb{M}_{n}$ be the space of $n \times n$ complex matrices. The Lieb-Thirring inequality [17] states that, for $0 \leq A, B \in \mathbb{M}_{n}$ and $p \geq 1$,

$$
\operatorname{Tr}\left(\left(B^{\frac{1}{2}} A B^{\frac{1}{2}}\right)^{p}\right) \leq \operatorname{Tr}\left(A^{p} B^{p}\right) .
$$

Let $A$ and $B$ be positive self-adjoint operators on a Hilbert space, and let $f$ be any increasing continuous function on $[0, \infty)$ such that $f(0)=0$ and $t \rightarrow f\left(e^{t}\right)$ is a convex function. Araki [2] shows a refinement of the Lieb-Thirring inequality as follows:

$$
\operatorname{Tr} f\left(\left(B^{\frac{1}{2}} A B^{\frac{1}{2}}\right)^{q}\right) \leq \operatorname{Tr} f\left(B^{\frac{q}{2}} A^{q} B^{\frac{q}{2}}\right) \quad \text { for all } q \geq 1 .
$$

Here the condition $f(0)=0$ ensures that the trace is well defined; that is, $\infty-\infty$ does not occur. Recently, Bourin and Lee [6] proved that if $f$ is increasing and $t \rightarrow f\left(e^{t}\right)$ is convex, then the inequality

$$
\operatorname{Tr} f\left(\left(B Z^{*} A Z B\right)^{q}\right) \leq \operatorname{Tr} f\left(B^{q} Z^{*} A^{q} Z B^{q}\right) \quad \text { for all } q \geq 1
$$

Copyright 2016 by the Tusi Mathematical Research Group.

Received Dec. 15, 2015; Accepted May 5, 2016.

2010 Mathematics Subject Classification. Primary 47A63; Secondary 46L52.

Keywords. Araki-Lieb-Thirring inequality, von Neumann algebra, $\tau$-measurable operator. 


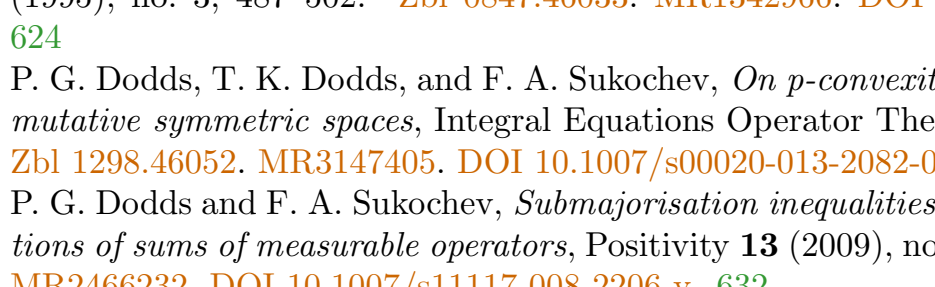

\title{
ON THE ARAKI-LIEB-THIRRING INEQUALITY IN THE SEMIFINITE VON NEUMANN ALGEBRA
}

\author{
YAZHOU HAN
}

Communicated by J.-C. Bourin

\begin{abstract}
This paper extends a recent matrix trace inequality of BourinLee to semifinite von Neumann algebras. This provides a generalization of the Lieb-Thirring-type inequality in von Neumann algebras due to Kosaki. Some new inequalities, even in the matrix case, are also given for the Heinz means.
\end{abstract}

\section{INTRODUCTION}

Let $\mathbb{M}_{n}$ be the space of $n \times n$ complex matrices. The Lieb-Thirring inequality [17] states that, for $0 \leq A, B \in \mathbb{M}_{n}$ and $p \geq 1$,

$$
\operatorname{Tr}\left(\left(B^{\frac{1}{2}} A B^{\frac{1}{2}}\right)^{p}\right) \leq \operatorname{Tr}\left(A^{p} B^{p}\right) .
$$

Let $A$ and $B$ be positive self-adjoint operators on a Hilbert space, and let $f$ be any increasing continuous function on $[0, \infty)$ such that $f(0)=0$ and $t \rightarrow f\left(e^{t}\right)$ is a convex function. Araki [2] shows a refinement of the Lieb-Thirring inequality as follows:

$$
\operatorname{Tr} f\left(\left(B^{\frac{1}{2}} A B^{\frac{1}{2}}\right)^{q}\right) \leq \operatorname{Tr} f\left(B^{\frac{q}{2}} A^{q} B^{\frac{q}{2}}\right) \quad \text { for all } q \geq 1 .
$$

Here the condition $f(0)=0$ ensures that the trace is well defined; that is, $\infty-\infty$ does not occur. Recently, Bourin and Lee [6] proved that if $f$ is increasing and $t \rightarrow f\left(e^{t}\right)$ is convex, then the inequality

$$
\operatorname{Tr} f\left(\left(B Z^{*} A Z B\right)^{q}\right) \leq \operatorname{Tr} f\left(B^{q} Z^{*} A^{q} Z B^{q}\right) \quad \text { for all } q \geq 1
$$

Copyright 2016 by the Tusi Mathematical Research Group.

Received Dec. 15, 2015; Accepted May 5, 2016.

2010 Mathematics Subject Classification. Primary 47A63; Secondary 46L52.

Keywords. Araki-Lieb-Thirring inequality, von Neumann algebra, $\tau$-measurable operator. 